\title{
TRANSPORT SIMULATION OF NEGATIVE MAGNETIC SHEAR DISCHARGES
}

\author{
by \\ H. ST JOHN, T.S. TAYLOR, Y.R. LIN-LIU, \\ and A.D. TURNBULL
}

This is a preprint of a paper to be presented at the Fifteenth International Conference on Plasma Physics and Controlled Nuclear Fusion Research, September 26, through October 1, 1994, in Seville, Spain, and to be published in the Proceedings.

Work supported by

U.S. Department of Energy

Contract No. DE-AC03-89ER51114

GENERAL ATOMICS PROJECT 3466 OCTOBER 1994 


\section{DISCLAIMER}

This report was prepared as an account of work sponsored by an agency of the United States Government. Neither the United States Government nor any agency thereof, nor any of their employees, make any warranty, express or implied, or assumes any legal liability or responsibility for the accuracy, completeness, or usefulness of any information, apparatus, product, or process disclosed, or represents that its use would not infringe privately owned rights. Reference herein to any specific commercial product, process, or service by trade name, trademark, manufacturer, or otherwise does not necessarily constitute or imply its endorsement, recommendation, or favoring by the United States Government or any agency thereof. The views and opinions of authors expressed herein do not necessarily state or reflect those of the United States Government or any agency thereof. 


\section{DISCLAIMER}

Portions of this document may be illegible in electronic image products. Images are produced from the best available original document. 
IAEA-CN-60/D-D-II-8

\section{ABSTRACT}

Simultaneous achievement of high energy confinement and high plasma beta could lead to an economically attractive compact tokamak fusion power plant [1]. High plasma performance regimes have been obtained in DIII-D with $H=\tau_{E} / \tau_{I T E R-89 P}=4$, terminated by MHD stability limits at high beta [2]. In DIII-D hollow current profiles, or second stable core (SSC) discharges, have been obtained with central beta values up to $44 \%$. Improved confinement in JET-PEP mode discharges has been obtained with hollow current profiles [3]. In the present work we present simulations which show that the hollow current profile can be maintained in quasi-steady state through a self-consistently determined combination of bootstrap current and neutral beam and rf current drive. Controllability of the $q$ profile is demonstrated by eliminating low $m / n$ mode number instabilities from these discharges by maintaining $q>1.5$ at all times,starting from appropriate initial conditions. At moderately high $\beta_{p}$, the bootstrap current can be a substantial fraction of the total current and the ability to maintain the proper total current density profile depends on the bootstrap current profile and the availability of suitable localized heating and current drive. In these simulations, we use electron cyclotron heating (ECH), ion cyclotron heating ( $\mathrm{ICH}$ ), and electron cyclotron and fast wave current drive (ECCD, FWCD). The ability to maintain the profiles is demonstrated using several energy transport models. Self-consistent transport simulations are used to model the SSC discharges using the ONETWO [4] transport code coupled to $r f$ heating and current drive packages FASTWAVE [5] and TORAY [6]. To accurately model inductive and driven current profile evolution, the additional source terms that arise in Faraday's law due to internal flux surface motion are included by coupling the transport calculations to a fixed boundary equilibrium code. To test the sensitivity of our results to the transport model used, we model DIII-D discharges using thermal conductivities consistent with improved confinement DIII-D discharges, and with the Rebut-Lallia-Watkins [7] model of energy transport. The density profiles in our simulations are chosen based on measured $\mathrm{VH}$-mode profiles. The modeling results indicate that simultaneous achievement of high confinement, $H>3.5$, high normalized beta, $\beta_{N}>5.0$ and high bootstrap fraction, is possible in DIII-D with these transport models. We have extended our simulations to demonstrate the applicability of these results to a compact fusion power plant. Using the RLW model of energy confinement it is shown that modest FWCD power applied near the hot ( $\sim 50 \mathrm{keV})$ center of such a power plant provides the necessary current drive control near the magnetic axis. Fast wave heating of the electrons and ions in the region of the high bootstrap current caused by the sharp turn on of the RLW model, allows some moderate control over the $q$ profile using a single, economically competitive fast wave heating system.

\section{INTRODUCTION}

In the results presented below the electron density and $Z_{\text {eff }}$ profile are held constant at prescribed initital values. The electron and ion temperature profiles 
together with Ampere's and Faraday's laws are evolved self consistently including the effects of flux surface motion. The electron and ion energy equations can both be written in the form

$$
\begin{aligned}
\frac{3}{2} n_{e, i} \frac{\partial T_{e, i}}{\partial t}+\frac{1}{I \rho} \frac{\partial}{\partial \rho}\left[I \rho\left(q_{e, i}+\frac{5}{2} T_{e, i} \Gamma_{e, i}\right)\right]= \\
S_{e, i}-\frac{5}{2} n_{e, i} T_{e, i} \frac{\partial(\ln I)}{\partial t}+\frac{d}{I^{\frac{5}{3}}} \frac{\partial}{\partial \rho}\left(\frac{3}{2} I^{\frac{5}{2}} n_{e, i} T_{e, i}\right)
\end{aligned}
$$

where $e, i$ means electron or (total) ion quantitites are to be taken. The source term $S_{e, i}$ includes the standard sources and sinks including neutral beam and radio frequency heating contributions. Faraday's law takes the flux surface average form

$$
\begin{aligned}
\frac{1}{F G(I \rho)^{2}} & {\left[\frac{\partial\left(F G I \rho B_{p 0}\right)}{\partial \rho}\right]-\frac{1}{I \rho} \frac{\partial}{\partial \rho}\left\{I \rho \left[d_{41} \frac{\partial n_{i}}{\partial \rho}+d_{42} \frac{\partial T_{e}}{\rho}+d_{43} \frac{\partial T_{i}}{\partial \rho}\right.\right.} \\
& \left.\left.+d_{44} \frac{\partial\left(F G I \rho B_{p 0}\right)}{\partial \rho}\right]\right\}-\frac{1}{I \rho} \frac{\partial\left(d B_{p 0}\right)}{\partial \rho} \\
& =-\frac{1}{I \rho} \frac{\partial}{\partial \rho}\left(\eta c\left\langle\frac{J_{a u x} \cdot \vec{B}}{B_{t 0}}\right\rangle\right)+\frac{1}{I \rho} \frac{\partial}{\partial \rho}\left[I \rho\left(D_{f}^{e}+D_{f}^{i}\right) \frac{\partial n_{f}}{\partial \rho}\right] \\
& +\frac{B_{p 0}}{I \rho} \frac{\partial}{\partial t}(\ln F G I \rho)+\frac{B_{p 0}}{I \rho} \frac{\partial d}{\partial \rho}
\end{aligned}
$$

where the terms in the gradient of $n_{i}, T_{e}, T_{i}$, and $D_{f}^{e}, D_{f}^{i}$ are related to the bootstrap current, $B_{p 0}$ is the poloidal magnetic field given by $B_{p 0}=\left(1 / R_{0}\right)(\partial \psi / \partial \rho)$ and the flux surface average geometry dependant factors are

$$
F=R_{0} B_{t 0} / f[\psi(\rho)] \quad, \quad G=\left\langle(\nabla \rho)^{2} R_{0}^{2} / R^{2}\right\rangle \quad, \quad I=F /\left\langle R_{0}^{2} / R^{2}\right\rangle
$$

The coefficients $F, G$, and $I$ are explicit functions of the radial flux coordinate rho (taken here as proportional to the square root of the toroidal flux) and implicit functions of time. In our calculations the time dependencies of $F, G$, and $H$ are assumed to be linear in between MHD equilibrium calculations. This time dependance, together with the apperance of the parameter $d$, which represents the speed of flux surfaces relative to the magnetic axis, give rise to the additional source terms in Eqs. (1) and (2). In the present work the equilibrium calculations are taken to be fixed plasma boundary up/down symmetric cases only (failure to assume up/down symmetry from the start was found to often cause vertical oscillations in the equilibrium calculations for the 
inverted q profile cases presented below). The function $f f^{\prime}$ which appears in the Grad-Shafranov (GS) equation is given by

$$
\left.f f^{\prime}=-\left\langle\frac{J_{\phi} R_{0}}{c R}\right\rangle+R_{0} P^{\prime}\right)\left(\frac{4 \pi R_{0} I}{F}\right)
$$

where the pressure gradient $P^{\prime}$ and flux surface average toroidal current density $\left\langle\frac{J_{\phi} R_{0}}{R}\right\rangle$ are determined from solution of Eqs. (1) and (2). Closure of the system of equations is achieved by taking Ohm's law in the form

$$
E_{0}(\rho)=I\left\langle\frac{\vec{E} \cdot \vec{B}}{B_{t 0}}\right\rangle=\eta I\left\langle\frac{\overrightarrow{J_{\text {ohm }}} \cdot \vec{B}}{B_{t 0}}\right\rangle
$$

$\eta$ is the parallel neoclassical resistivity, $R_{0}, B_{t 0}$ are reference major radius and magnetic field values, and $E_{0}$ is the ohmic electric field (in steady state $E_{0}$ is related to the loop voltage by $V_{\text {loop }}=2 \pi R_{0} E_{0}$ ). The parallel ohmic current is given in terms of the total, bootstrap and auxiliary driven currents by

$$
\left\langle\frac{\vec{J}_{o h m} \cdot \vec{B}}{B_{t 0}}\right\rangle=\left\langle\frac{\vec{J} \cdot \vec{B}}{B_{t 0}}\right\rangle-\left\langle\frac{\vec{J}_{b o o t} \cdot \vec{B}}{B_{t 0}}\right\rangle-\left\langle\frac{\vec{J}_{a u x} \cdot \vec{B}}{B_{t 0}}\right\rangle \text {. }
$$

Where the total parallel current is determined from Ampere's law, the auxiliary driven current is determined from beam and rf current drive models, and the bootstrap is taken as the small inverse aspect ratio, collisional model of Hirshman [11]. In the flux surface average formulation presented here the total toroidal current inside a flux surface and the safety factor are

$$
I(\rho)=\frac{c}{2} G I \rho B_{p 0} \quad, \quad q=\frac{c}{2} \frac{B_{t 0}}{R_{0}}\left[\frac{G I \rho^{2}}{I(\rho)}\right] . \quad(7 a, b)
$$

\section{DIII-D SIMULATIONS}

The up/down symmetric, fixed boundary, $\left(\kappa=2.1, \delta=0.8, R_{a}=1.9 \mathrm{~m}\right)$ DIII-D type discharge shape used in the analysis is shown in Fig. 1 with the initial and final (near steady state) flux surfaces generated using the Hsieh [10] model of confinement. The initial MHD equilibrium configuration is given by the solid contours in the figure and was the same for all three confinement models presented below. The final configuration, indicated by dashed lines in the figure, in addition to being an MHD equilibrium state, is also a transport equilibrium state where the temperatures are constant in time and the electric field, $E_{0}$, is constant in rho. The rearrangement of the flux surfaces is typical of the cases presented here and is indicative of the effects of including transport calculations in our simulations. We have observed that failure to evolve the equilibrium with the transport leads to final states which do not satisfy the GS equation and hence are states that could not be achieved experimentally. The geometric factors $F, G, I$, appearing in Eqs. (1) and (2) can vary substantially 


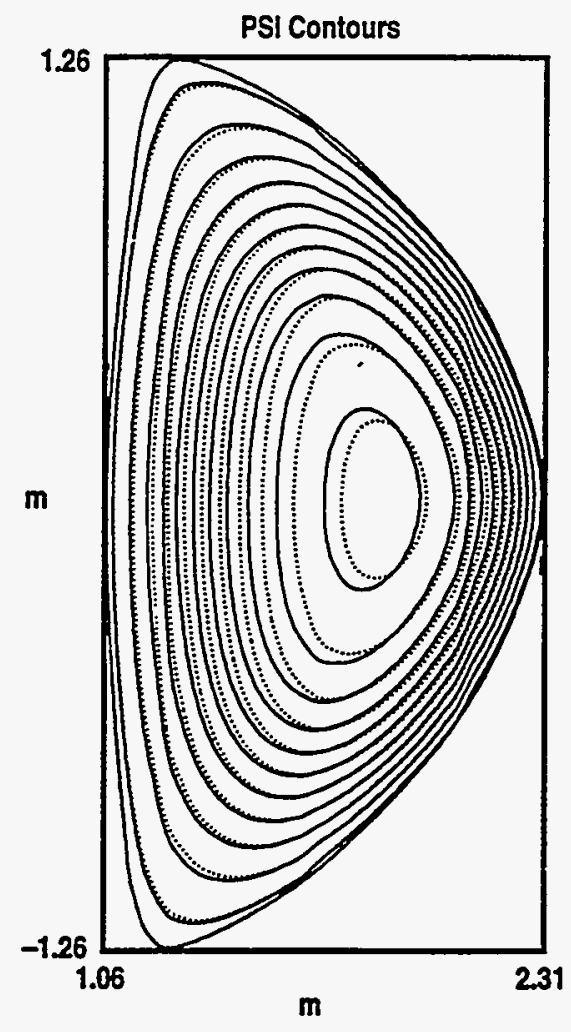

Fig. 1. Motion of flux surfaces during the simulation. Solid contours represent starting configuration, dashed contours represent the final MHD/transport equilibrium configuration for the Hsieh confinement model.

during the evolution. For the case illustrated in Fig. 1 a maximum relative change of 30 percent occured in the $G$ function, near the magnetic axis, during the 12 second simulation of the evolving plasma. The changes in $F$ are more benign, amounting to about 4 percent near the axis, while the factor $I$ changed a maximum of 8 percent, also near the axis. The additional sources in Eqs. (1) and (2) amounted to a maximum of 4 and 9 percent of the RHS of these equations respectively,due to the dominance of auxiliary heating and current drive. Much larger variations than these can be expected during the active shaping of the hollow current profile (which could be achieved by elongating the plasma and/or using FW current to decrease the beam driven current near the axis). The present paper does not address these startup issues.

A VH-mode type electron density profile, with a line average density of $5.80 \times 10^{19} / \mathrm{m}^{3}$ was used to satisfy the Greenwald [8] limit. Three different energy confinement models, representative of Bohm, gyro-Bohm, and nonlinear grad $T_{e}$ dependent diffusion were used in the calculations. The simplest (Bohm) model, consistent with observed transport in DIII-D, has an electron thermal diffusivity given by INTOR scaling

$$
\chi_{e}=\frac{5.0 \times 10^{19}}{n_{e}\left[1-0.8(\rho / a)^{2}\right]} \quad\left(\frac{\mathrm{m}^{2}}{\mathrm{~s}}\right)
$$


The ion conductivity was taken as $\chi_{i}=2.6 \chi_{i, n e o}$ where the factor of 2.6 was choosen to yield temperature profiles that are representative of $\mathrm{VH}$-mode and high confinement $\mathrm{H}$-mode discharges [8]. A representative gyro-Bohm case is the RLW model of confinement [7] which features a large and rapid increase in the diffusivity when the magnetic shear becomes positive. This leads to very steep temperature gradients for SSC discharges as is shown below. Finally, a recently developed model based on analysis of $\mathrm{L}$-mode data by Hsieh [10], is of the nonlinear grad $T_{e}$ variety investigated by Dnestrovskij [13] and indicates that the electron and ion diffusivities are well fit by the formulae

$$
\chi_{e}=\chi_{\mathrm{Hsieh}}+\chi_{e, \text { neo }} \quad \chi_{i}=C_{i} \chi_{\mathrm{Hsieh}}+\chi_{i, \text { neo }}
$$

where

$$
\chi_{\mathrm{Hsieh}}=C_{e} \frac{n_{e} T_{e}}{B_{p}^{2}}\left(\frac{r^{3}}{R}\right)\left(\frac{1}{T_{e}} \frac{\partial T_{e}}{\partial \rho}\right)^{2}
$$

$C_{e}$ and $C_{i}$ are adjustable constants. The value used for $C_{e}$ is approximately 0.1 times the value determined by fitting to experimental data in $\mathrm{L}$-mode discharges and was chosen to give the representative VH-mode temperature profiles.

The results of the combined equilibrium and transport simulations is summarized in Table I. The three confinement models for DIII-D each relied on

Table 1

Results for the DIII-D and Plower Plant Simulations

\begin{tabular}{lllll}
\hline & INTOR & RLW & Hsieh & $\begin{array}{l}\text { Power } \\
\text { Plant }\end{array}$ \\
\hline$\beta_{N}$ & 5.7 & 5.2 & 5.4 & 6.2 \\
$\beta_{P}$ & 2.52 & 2.50 & 2.45 & 3.3 \\
$H$ & 3.6 & 3.5 & 3.8 & 4.1 \\
$I_{P}(\mathrm{kA})$ & 1,600 & 1,600 & 1,600 & 11,500 \\
$I_{\text {boot }}$ & 1,070 & 975 & 1,170 & 11,200 \\
$I_{R F}$ & 309 & 349 & 286 & 337 \\
$I_{\text {beam }}$ & 172 & 266 & 181 & 0 \\
$I_{\text {ohm }}$ & 49 & 9 & -39 & -87 \\
$P_{F W}(\mathrm{MW})$ & 6.5 & 6.5 & 6.5 & 27 \\
$P_{E C H}$ & 7.0 & 8.0 & 8.0 & 0 \\
$P_{N B I}$ & 6.5 & 6.5 & 4.5 & 0 \\
$\gamma^{*}$ & 0.04 & 0.04 & 0.03 & 0.45 \\
$l_{i}$ & 0.57 & 0.84 & 0.64 & 0.86 \\
$\mathrm{~W}(\mathrm{MJ})$ & 3.8 & 3.6 & 3.6 & 912 \\
$q_{\text {min }}$ & 2.57 & 1.86 & 2.54 & 2.06 \\
\hline${ }^{*} \gamma=\overline{n_{e}} I_{R F} R_{0} / P_{R F}, A / W / M^{2} / 10^{20}$ & &
\end{tabular}


6.5 MW of FW heating deposited near the magnetic axis, using two oppositely directed FW channels so as to drive no current. The necessary current near the magnetic axis is supplied by the neutral beam. By changing the FW power balance in the two channels it is possible to modulate the current density near the magnetic axis, providing the necessary control of $q(0)$. Seven or eight MW of ECH power, deposited near $\rho=0.5$ was used to to maintain the inverted $q$ profile for these cases.

The hollow $q$ profiles for the three confinement models are shown in Fig. 2, and the corresponding steady state total toroidal current profiles are given in Fig. 3. The electron and ion diffusivities are shown in Fig. 4 for the three confinement models. Each of the models yields a rapid rise in the diffusivity near the magnetic axis due to the relatively small current density and hence poloidal magnetic field. The diffusivity of the RLW model suddenly increases by more than an order of magnitude due to the step function $\nabla q$ dependance of that model. As indicated in Fig. 4 we have taken the neoclassical background for the electron diffusivity in the RLW and Hsieh model to be equal to the ion neoclassical diffusivity. We find that such an adjustment is necessary in

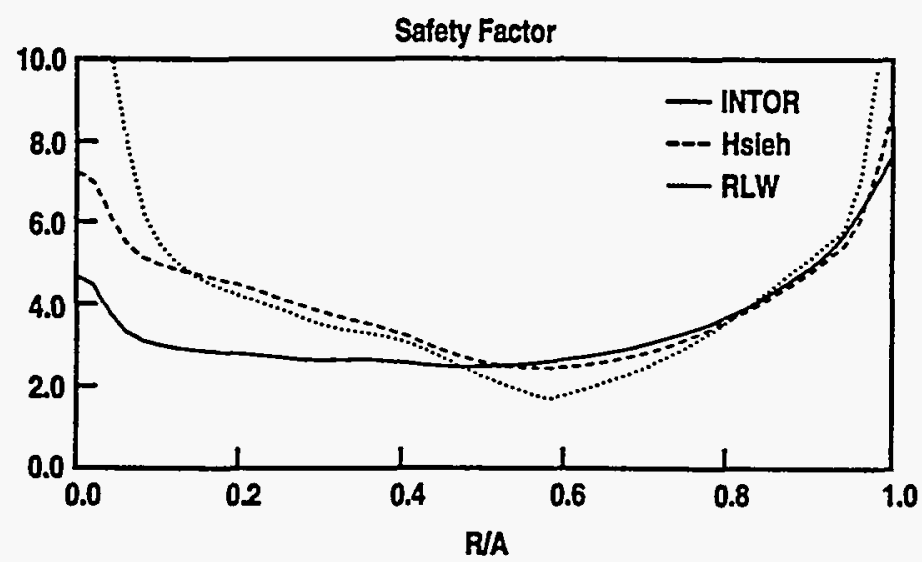

Fig. 2. The safety factor profile for the three energy confinement models discussed in the text.

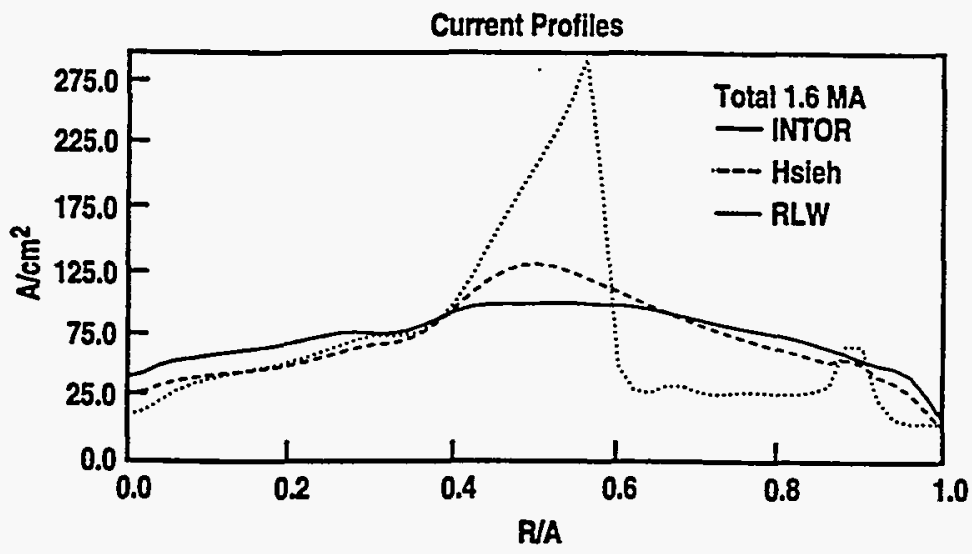

Fig. 3. The current profiles associated with the $q$ profiles of Fig. 2. 

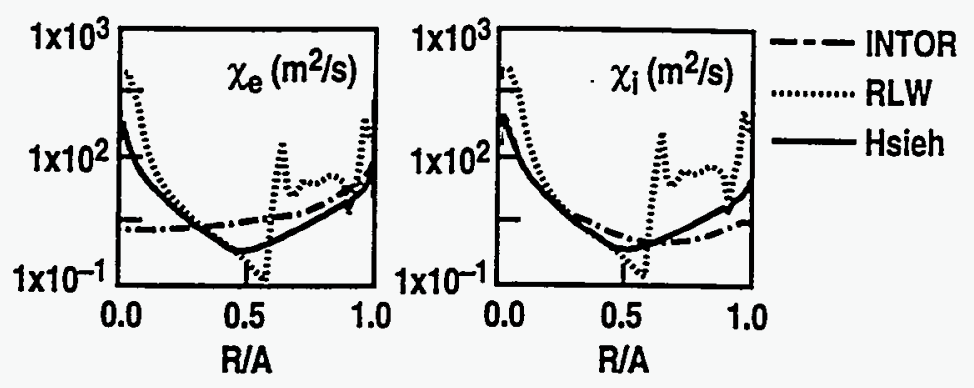

Fig. 4. The electron and ion thermal conductivities for the INTOR, Hsieh, and RLW confinement models.

order to avoid hollow electron temperature profiles during simulation of off axis ECH heated discharges. Evolution of the electron and ion energy using Eq. (1), magnetic and electric fields using Eqs. (2) to (6) and the GS equation, and the assumed fixed particle densitites yields the electron and ion temperatures shown in Fig. 5 for the three models. The RLW model is seen to produce a very sharp temperature gradient due to the rapid rise in the electron and ion thermal conductivities as we pass from the region of negative to positive magnetic shear, see Fig. 2. Such steep temperature gradients have been observed experimentally, see for example the reversed magnetic shear PEP mode results of Hugon [3].

A characteristic feature of the RLW model is that it produces lower values of minimum $\mathrm{q}$ than the other confinement models examined here. Due to the large and rapid rise of the bootstrap current as the region of reversed magnetic shear is crossed, there is an initial rapid rise of the total enclosed current. This leads to a sharp decrease in $q$ by way of Eq. (7b). One could move the value of rho at which the minimum q occurs closer to the magnetic axis, thereby moving the temperature gradient and associated peak in the bootstrap current into regions of smaller volume. This decreases the total enclosed current and raises the minimum value of $q$. However the total bootstrap current is thereby also lowered so that larger amounts of ohmic and/or rf current will be required for a given total plasma current. The ohmic bootstrap, beam, and ECCD contributions to the total plasma current profile for the RLW case is shown in Fig. 6. Note the ECCD current required near the peak of the bootstrap current to bring the total
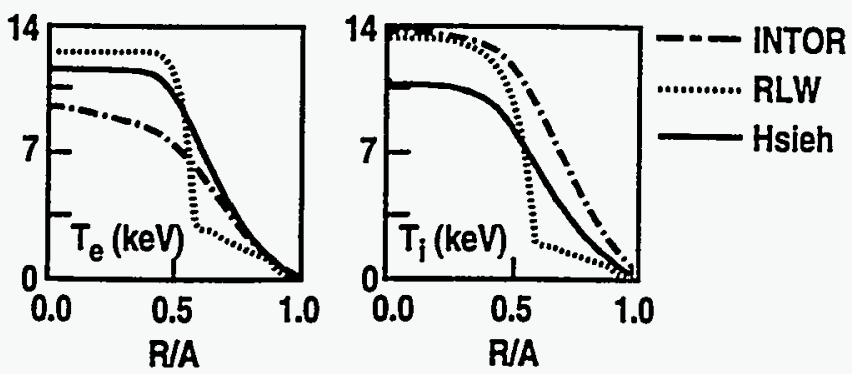

Fig. 5. Final steady-state electron and ion temperatures for the confinement models of Fig. 4. 


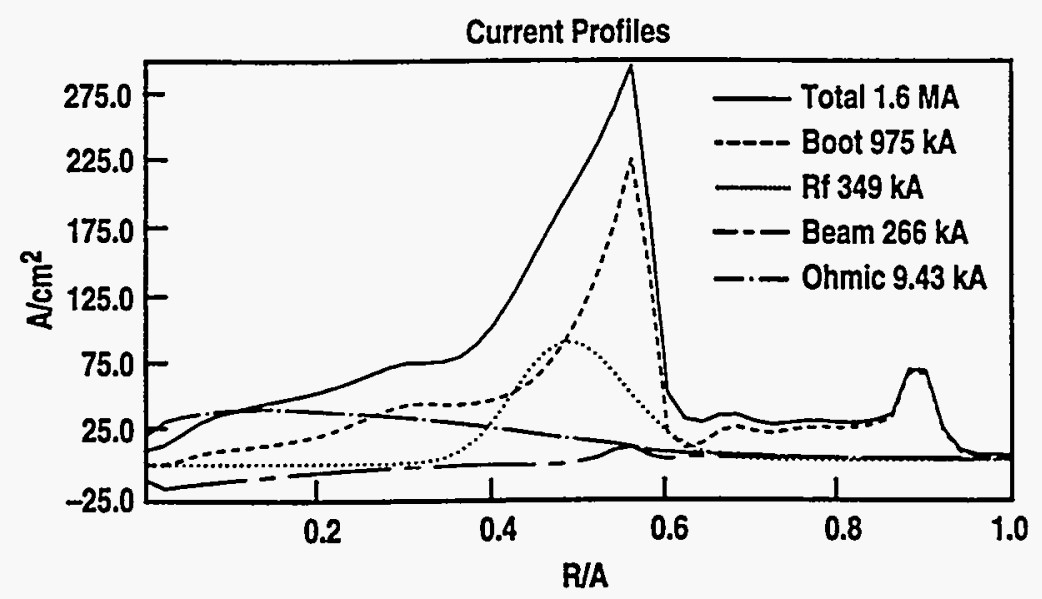

Fig. 6. The total, bootstrap, ECCD, beam, and ohmic current profiles for the DIII-D RLW simulation.

ohmic current down to near zero. The secondary peak in the RLW bootstrap and total current profile, centered near $\rho=0.9$ in Fig. 6 , is due to the boundary condition used for Eq. (1) at the plasma edge. This boundary condition, which specifies the value of $T_{e}$ (and $T_{i}$ ) was set to the typical DIII-D value of $0.1 \mathrm{keV}$. Raising this value to $0.3 \mathrm{keV}$ would eliminate the secondary current peak.

The INTOR, RLW and Hsieh models were each evolved from the same initital state with a fixed plasma current. As a consequence we find that the near steady state RLW model has a minimum $q$ value that drops below 2 and thus a region of instability not present in the INTOR and Hsieh models is introduced. If the constraint on the total plasma current is relaxed it is possible to find solutions with a minimum $q$ value above 2 . An example relevant for power plant conditions is given in the next section. With the exception of the RLW model these discharges are stable to $n=1,2$. A detailed account of the stability properties of such discharges is given by Turnbull [12].

As summarized in Table I we have identified attractive second stable core scenarios applicable to DIII-D operation, with high confinement, high beta, and with a large, properly aligned, bootstrap current fraction. The simultaneous achievement of these properties experimentally is part of the DIII-D Advanced Tokamak program. According to our simulations the planned availability of $8 \mathrm{MW} \mathrm{FW}$ and $10 \mathrm{MW}$ ECCH will be sufficient to achieve these goals.

\section{COMPACT POWER PLANT SCENARIO}

The high core temperatures that will be present in fusion power plants can be advantageously used in FWCD scenarios. Included in Table 1 are the parameters of a compact scaled up DIII-D power plant (with the same elongation and triangularity as DIII-D and a major radius of $5.9 \mathrm{~m}$ ). Using a fixed electron density profile scaled up from DIII-D VH-mode density profiles (at about 1.1 times the Greenwald limit) and modeling energy confinement with the RLW model we have generated a reactor scenario which is close to $100 \%$ bootstrap current driven. Using $6.75 \mathrm{MW}$ of $\mathrm{FW}$ power at $20 \mathrm{MHz}$ it is possible with 
off-axis ECH to drive $20 \mathrm{~A} / \mathrm{cm}^{2}$ of $\mathrm{FW}$ current at the magnetic axis in such a reactor, see Fig. 7 where the current profile and its constituent bootstrap, FW and ohmic contributions are shown. The bootstrap current is of sufficient magnitude to supply essentially all of the plasma current (Table 1). Electron and ion temperature profiles due to $\mathrm{FW}$ heating at four power levels are show in Fig. 8. The FW heating at $140 \mathrm{MHz}$ (about $1 / 3$ the power absorbed by electrons and $2 / 3$ absorbed by ions) is used to shape the temperature profile as indicated in the figure. The resulting modulation of the bootstrap current gives rise to the safety factor and current profiles shown in Figs. 9 and 10. By sweeping the off axis FW power from zero to $50 \mathrm{MW}$ the minimum $q$ value rises from 1.68 to 2.13 . However for more than about $20 \mathrm{MW}$ of FW heating the current profile becomes quite narrow near $q_{\min }$ and a shallow second minimum in $q$ develops, see the $50 \mathrm{MW}$ cases in Figs. 9 and 10. Hence we may expect that an approximate 20 percent variation in $q_{\min }$ can be achieved using this profile method.

\section{CONCLUSION}

Improved confinement discharges have properties such as relatively low internal inductance, high edge pressure gradients and high edge bootstrap currents that are unfavorable for high beta. However using negative central shear with the minimum $q$ value slightly above rational values leads to stable operating regimes. The model discharges presented here can be stabilized at high beta by having a wall sufficiently close to the plasma. Attractive DIII-D second stable core scenarios have been identified which should be confirmable experimentally in the near furture. Although the details of the results depend on the energy confinement model used, the INTOR, Hsieh, and RLW models all confirm that it should be possible to maintain a second stable core with high confinement, $\mathrm{H}$ greater than 3.5, and high normalized beta, $\beta_{N} \geq 5$. The results do not require unresonable amounts of fast wave and $\mathrm{ECH}$ power in order to be achieved. After initial establishment of a hollow current profile it is still necessary to evolve the equilibrium and transport equations simultaneously in order to ensure that

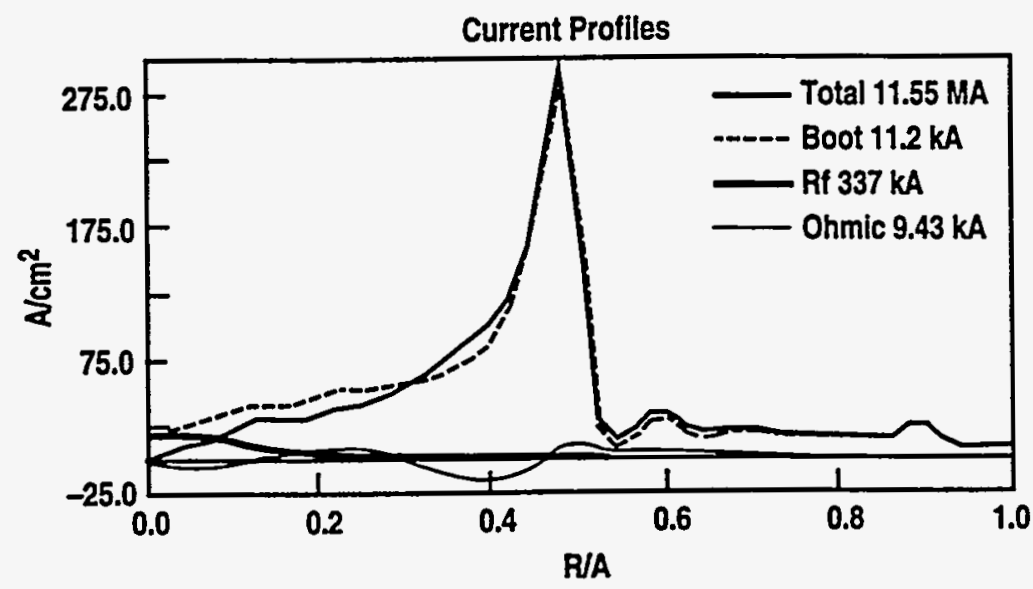

Fig. 7. The total, bootstrap, FWCD, and Ohmic current profiles for the compact power plant. 

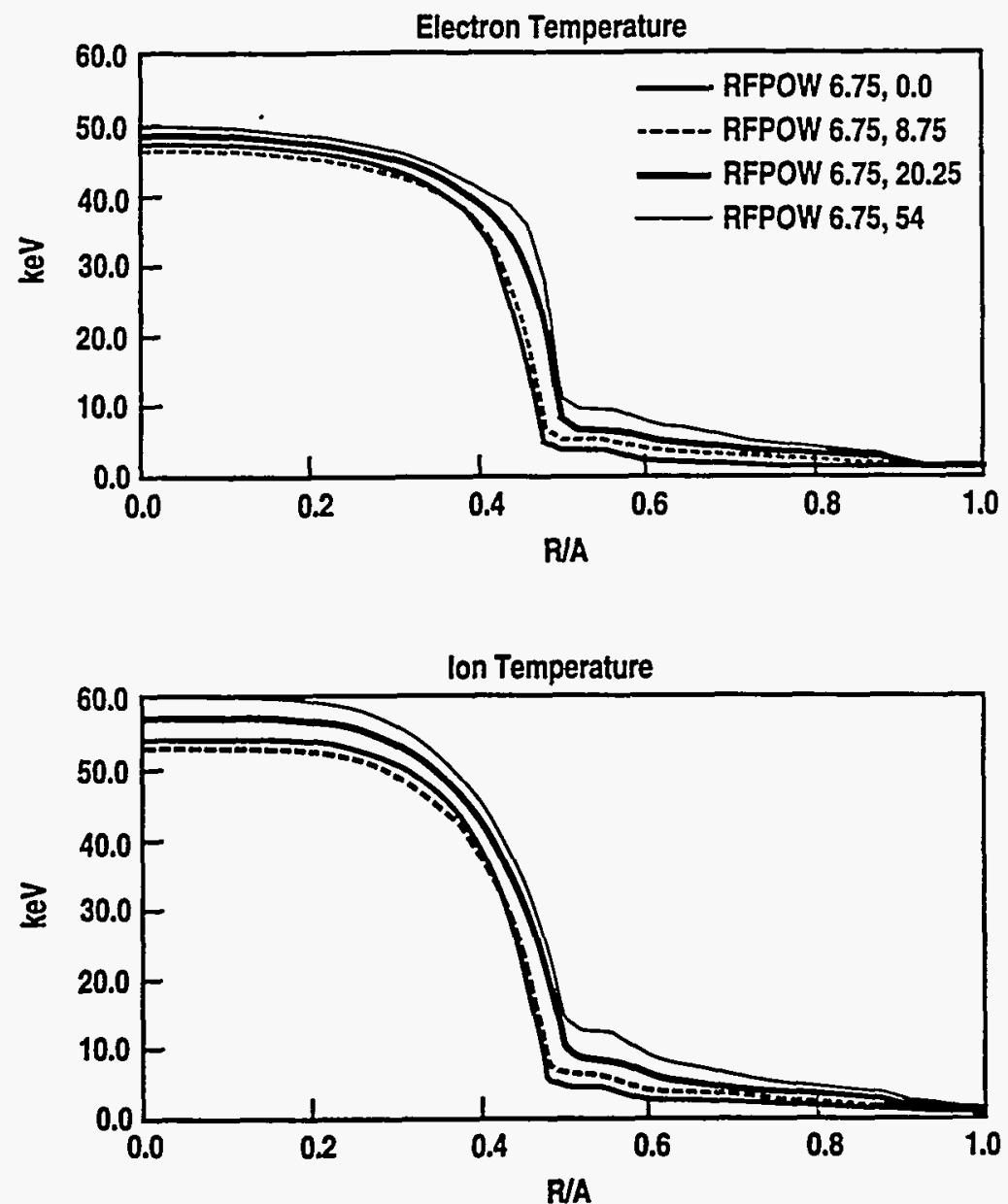

Fig. 8. The electron and ion temperatures at four different $F W$ heating power levels.

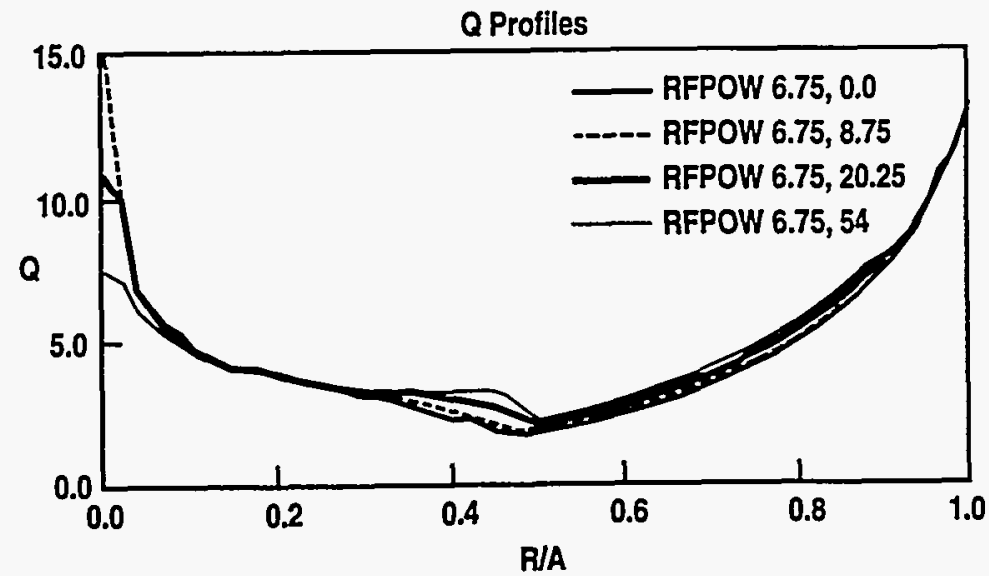

Fig. 9. The safety factor profile at the four power levels corresponding to Fig. 8. 


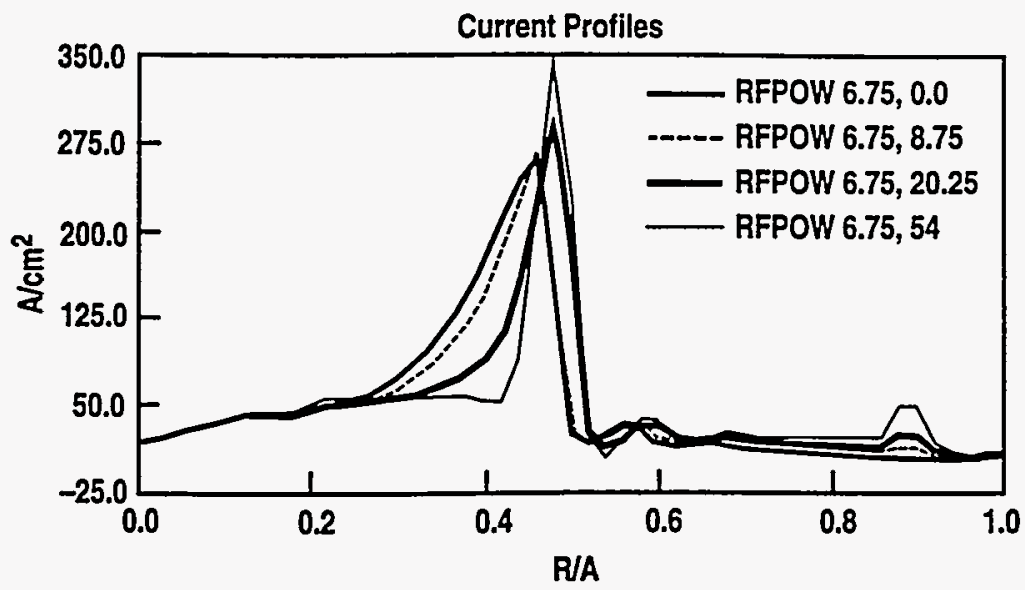

Fig. 10. The total current profile at the four power levels of Fig. 8.

the slowly evolving equilibrium state satisfies the GS equation. Even for fixed boundary equilibria the rearrangement of the internal flux surfaces is sufficient to change the equilibrium and hence stability properties of the discharge.

Scaled up DIII-D compact power plant scenarios are still under investigation. At present we have found a reversed shear, high performance scenario with essentially $100 \%$ bootstrap current. It appears that the necessary control over the $q$ profile in such reactors can be achieved using only fast wave heating and small amounts of current drive, given that such a configuration was established initially.

\section{REFERENCES}

[1] PERKINS, L.J., GALAMBOS, J.D., HANEY, S.W., MADREKAS, J., "Commercial Tokamak Reactor Potential With Advanced Tokamak Operation," submitted to Nucl. Fusion, March 1994.

[2] STRAIT, E.J., "Beta-Limiting Instabilities in DIII-D Discharges with Large Bootstrap Current," in Proc. 20th Euro. Conf. on Contr. Fusion and Plasma Heating, Lisbon, Portugal,1993 (European Physical Society, Petit-Lancy, Switzerland,1993) Vol. 17C, Part I ,p. 211.

[3] HUGON, etal., Nucl. Fusion 32 (1992) 33.

[4] ST JOHN, H., etal., op. cit., Vol. 1, p. 99.

[5] CHIU, S.C., etal., Nucl. Fusion 29 (1989) 2175.

[6] MATSUDA, K., IEEE Trans. Plasma Sci. 17, (1989) 6.

[7] REBUT, P., et al., Plasma Phys. and Contr. Nucl Fusion, Nice,1988 (IAEA, Vienna,1989).

[8] GREENWALD, M., etal., Nucl. Fusion 28 (1988) 2199.

[9] TAYLOR, T.S., etal., Plasma Phys. and Contr. Nucl. Fusion Research 1992, Würzburg (IAEA, Vienna, 1993) Vol. 1, p. 167. 
[10] HSIEH, C.L., et al., Bull. Am. Phys. Soc. 38 (1993) 2065.

[11] HIRSHMAN, S., Phys. Fluids 21 (1978) 8.

[12] TURNBULL, A.D., etal., "High Beta and Enhanced Confinement in a Second Stable Core VH-Mode Advanced Tokamak," submitted to Phys. Rev. Lett.

[13] DNESTROVSKIJ, Yu.M., et al., in Proc. of 18th Euro. Conf. on Contr. Fusion and Plasma Phys., Berlin, Germany, 1991 (European Physical Society, Petit-Lancy, Switzerland, 1991) Vol. 1, p. 413.

\section{ACKNOWLEDGMENT}

This is a report of work supported by the U.S. Department of Energy under Contract No. DE-AC03-89ER51114. 